\title{
Features of highly structured equatorial plasma irregularities deduced from CHAMP observations
}

\author{
C. Xiong ${ }^{1,2}$, H. Lühr ${ }^{1}$, S. Y. Ma ${ }^{2}$, C. Stolle ${ }^{3}$, and B. G. Fejer ${ }^{4}$ \\ ${ }^{1}$ Helmholtz Centre Potsdam, GFZ German Research Centre for Geosciences, Telegrafenberg, 14473, Potsdam, Germany \\ ${ }^{2}$ Department of Space Physics, College of Electronic Information, Wuhan University, Wuhan 430079, China \\ ${ }^{3}$ National Space Center, Technical University of Denmark, 2100 Copenhagen, Denmark \\ ${ }^{4}$ Center for Atmospheric and Space Science, Utah State University, Logan UT 84322, USA
}

Correspondence to: C. Xiong (xiongchao@whu.edu.cn)

Received: 18 March 2011 - Revised: 2 August 2012 - Accepted: 3 August 2012 - Published: 24 August 2012

\begin{abstract}
In this study five years of CHAMP (Challenging Mini-satellite Payload) fluxgate magnetometer (FGM) data is used to investigate the characteristics of Equatorial Plasma Bubbles (EPBs). We filtered the FGM data by using bandpasses with four different cut-off periods to get the EPBs with different maximum spatial scale sizes in the meridional plane ranging from $76-608 \mathrm{~km}$. Associated with the EPB observations at about $400 \mathrm{~km}$, the typical altitude of CHAMP during the year 2000-2005, we also investigate the postsunset equatorial vertical plasma drift data from ROCSAT1 (Republic of China Satellite 1). Since the height of the F-layer is highly correlated with the vertical plasma drift and solar flux, we sorted the ROCSAT- 1 data into different groups by F10.7. From the integrated vertical drift we have estimated the post-sunset uplift of the ionosphere. By comparing the properties of EPB occurrence for different scale sizes with the global distribution of plasma vertical uplift, we have found that EPBs reaching higher altitudes are more structured than those which are sampled by CHAMP near the top side of the depleted fluxtube. Such a result is in accord with 3-D model simulations (Aveiro and Hysell, 2010). Small-scale EPB structures are observed by CHAMP when the irregularities reach apex heights of $800 \mathrm{~km}$ and more. Such events are encountered primarily in the Brazilian sector during the months around November, when the post-sunset vertical plasma drift is high.
\end{abstract}

Keywords. Ionosphere (Equatorial ionosphere; Ionospheric irregularities)

\section{Introduction}

The term equatorial plasma bubble (EPB) stands for a localized region of plasma density depletion in the nighttime lowlatitude F-region (e.g. Kelley, 2009, Sect. 4), which is believed to be generated at the bottom side of the F-region when the ionosphere is unstable to the growth of the RayleighTaylor (R-T) instability (Kelley, 2009, Sect. 4). During their evolution EPBs rise upward and increase in latitudinal extend (Whalen, 2000). The global distribution of EPBs has been studied for decades, and a typical seasonal/longitudinal (S/L) distribution was deduced from both ground observation and satellite measurements (Sobral et al., 1999; Makela and Kelley, 2003; Burke et al., 2004). Their studies have shown that the EPB occurrence shows a seasonal and longitudinal dependence. During June (December) solstice the occurrence rate reaches its highest values in the African and Pacific (South America and Atlantic) regions. During equinoxes the occurrence rate is generally higher, and the longitudinal variation is smaller than that of solstices. Huang et al. (2001, 2002) and Burke et al. (2004) surveyed the Defense Meteorological Satellite Program (DMSP) $(840 \mathrm{~km}$ altitude) data and got nearly the same S/L distribution as described above. Su et al. (2006, 2007), using Formosa Satellite-1/Republic of China Satellite-1 (FORMOSAT-1/ROCSAT-1, $600 \mathrm{~km}$ altitude), obtained a similar S/L distribution of EPBs around the solar maximum in 2001. Xiong et al. (2010) have confirmed from both CHAMP and GRACE (Gravity Recovery and Climate Experiment) electron density data that the most severe occurrence rate of EPBs is observed above Africa and the Pacific Ocean (the South America) around June (December) solstice months. 
An important aspect of EPBs is their latitudinal distribution. Stolle et al. (2006) found a latitudinal distribution of EPB occurrences detected by CHAMP at $400 \mathrm{~km}$ altitude that peaks at $\pm 9.5^{\circ}$ magnetic latitude (Mlat) with a standard deviation of $4.5^{\circ}$ in both hemispheres. This is in apparent contrast with EPB occurrence rates deduced from ROCSAT-1 $(\sim 600 \mathrm{~km})$ and DMSP $(840 \mathrm{~km})$ observations, which peak at the geomagnetic equator and decrease symmetrically to $\pm 20^{\circ}$ magnetic latitude (Burke et al., 2004). Xiong et al. (2010) have investigated the EPB from electron density data obtained by the CHAMP and GRACE satellites. Their results have shown that at CHAMP altitude $(\sim 400 \mathrm{~km})$, the EPB occurrence peaks on average at $-8.74^{\circ}$ and $9.47^{\circ}$ Mlat in the Southern and Northern Hemisphere and at $-6.96^{\circ}$ and $8.25^{\circ}$ Mlat at GRACE altitude $(\sim 500 \mathrm{~km})$, respectively. Comparing the results from the two satellite missions, it is observed that at higher altitude the EPBs appear closer to the magnetic equator. This was interpreted by Xiong et al. (2010) as an indication that the plasma depletion associated with an EPB evolves along magnetic fluxtubes. Under these conditions the apex height of EPBs can be estimated, and it is found in most cases to range around $600 \mathrm{~km}$, reconciling the different latitudes of occurrence peaks from CHAMP and ROCSAT- 1 observations.

By operating the ALTAIR radar, which is located in the Kwajalein Atoll, Marshal Islands at a magnetic dip latitude of $4.3^{\circ} \mathrm{N}$, in a latitude scan incoherent scatter mode, Tsunoda (1980) present direct evidence that plasma bubbles are indeed magnetic field aligned and extend over at least $10^{\circ}$ of magnetic latitude. Furthermore, Makela et al. (2005) present two examples using data collected by a ground-based imaging system on Haleakala Volcano on Maui, Hawaii, and insitu density plasma density measurements taken at $600 \mathrm{~km}$ by ROCSAT- 1 . In their results the composite images were mapped along the magnetic field lines to $600 \mathrm{~km}$ altitude. Excellent agreement was found between the location of the depletions estimated by the composite imaging technique and the ROCSAT-1 data, even in the conjugate hemisphere. Yokoyama et al. (2007) reported a concurrent observation of radar echo and plasma irregularities along the same meridian in opposite hemispheres but at magnetically conjugate locations.

It is well know that EPBs can have scale sizes from a few meters to thousands of $\mathrm{km}$, due to different generation and developing EPB conditions (Zargham and Seyler, 1989; Hysell and Seyler, 1998; Hysell, 2000). Singh et al. (1997) presented some examples from Atmosphere Explorer E (AE-E) data showing plasma bubbles developing from wavy ion density structures in the bottom side F-layer. The wavy structures mostly had east-west wave lengths of $150-880 \mathrm{~km}$ and the ionization trough in the wavy structures later broke up into either multiple bubble patches or a single bubble. In the multiple bubble patches, intra-bubble spacing varied from 55 to $140 \mathrm{~km}$. In a fully developed equatorial spread $\mathrm{F}$ case, east-west wavelengths from $690 \mathrm{~km}$ down to about $0.5 \mathrm{~km}$ were present simultaneously. Xiong et al. (2010), by analyzing the electron density data of CHAMP and GRACE satellites, found that both satellites may detect more than one electron depletion regions along one meridional passage over the low latitude regions. Stolle et al. (2006) have used high-resolution CHAMP magnetic field readings $(50 \mathrm{~Hz})$ to investigate the fine structure of detected EPB events in the meridional plane. Their presented event exhibited structures as small as about $50 \mathrm{~m}$. Narrowest features were commonly observed at the poleward boundaries of a series of EPBs.

The close relationship between EPB occurrence rate and the amplitude of the vertical plasma drift, or the layer height, was shown empirically in several studies (Farley et al., 1970; Fejer et al., 1999; Stolle et al., 2008). Fejer et al. (2008) used ROCSAT-1 data to develop a model for the vertical plasma drift, which was used by Stolle et al. (2008) for their analysis. Jayachandran et al. (1993) investigated HF Doppler radar and ionosonde measurements in India, and stated that the height of the F-layer, determined by the evening vertical plasma drift, is the deciding factor for an EPB onset. They also stated that the R-T growth rate maximizes at the time of the peak height rather than at the time of peak velocity. Stolle et al. (2008) and Su et al. (2008) have confirmed that the EPB occurrence rate has a higher correlation with the integrated vertical plasma drift velocity than with the peak value of the vertical plasma drift velocity during the pre-reversal enhancement (PRE).

Questions we want to address are the spatial scale distribution of EPBs, and what are the characteristics of the different scale sizes in the meridional plane, and how do they depend on prevailing conditions? A near-polar orbit satellite such as CHAMP provides us excellent opportunities to study the latitudinal structures of EPBs. For this paper we have used the first five years of CHAMP magnetic field data and ROCSAT1 ion drift data to verify that the high vertical plasma drift plays an important role for the small-scale size EPB to rise to the CHAMP altitude (about $400 \mathrm{~km}$ ). Results obtained provide first observational evidence that some EPBs are highly structured at the bottom side.

\section{Data}

The CHAMP satellite was launched on 15 July 2000 into a circular, near-polar (inclination $=87.3^{\circ}$ ) orbit with an initial altitude of about $450 \mathrm{~km}$. Its local time changes by about $5.5 \mathrm{~min}$ per day, sweeping all time zones in 131 days (Reigber et al., 2002). After 3718 days in orbit, it reentered the Earth's thermosphere and burned up on 19 September 2010. In this paper we use primarily the fluxgate magnetometer (FGM) data to investigate EPBs by their magnetic signatures. In order to avoid false detections due to storm-related magnetic pulsation effects, we have selected quiet to moderately disturbance times as indicated by $K_{p} \leq 3.5$. In addition plasma density readings from the Planar Langmuir probe 
Table 1. The low-pass and high-pass filters with different cut-off periods used for detecting different scale size EPBs.

\begin{tabular}{crr}
\hline $\begin{array}{c}\text { Low-pass } \\
(\mathrm{LP}) \text { period }\end{array}$ & $\begin{array}{r}\text { High-pass } \\
(\mathrm{HP}) \text { period }\end{array}$ & $\begin{array}{r}\text { Scale size } \\
(\mathrm{km})\end{array}$ \\
\hline $8 \mathrm{~s}$ & $40 \mathrm{~s}$ & $120-608$ \\
$4 \mathrm{~s}$ & $20 \mathrm{~s}$ & $60-304$ \\
$2 \mathrm{~s}$ & $10 \mathrm{~s}$ & $30-152$ \\
$1 \mathrm{~s}$ & $5 \mathrm{~s}$ & $15-76$ \\
\hline
\end{tabular}

(PLP) data are also taken into account. The sparse sampling, once per $15 \mathrm{~s}$, however, provides only a coarse overview. Here we consider data from the more active $5 \mathrm{yr}$ from $\mathrm{Au}-$ gust 2000 to July 2005.

For the EPB detection approach from FGM data (vector magnetic field data at high resolution), we follow the approach introduced by Stolle et al. (2006). In order to identify the small magnetic signatures of EPBs it is necessary to subtract the main field and the large-scale magnetospheric fields from the original readings. The residual field is transformed into Mean-Field-Aligned (MFA) coordinates, comprising two transverse components and one field-aligned. Only the latter, parallel component $B \mu$, is of interest here. As in Fig. 3 of Stolle et al. (2006), the parallel component, $B \mu$, is first filtered with a band pass filter and then rectified. After that a threshold is set at $\mathrm{LU}=0.20 \mathrm{nT}$. For an EPB to be detected, the rectified amplitude has to reach above LU for several successive readings. In this paper we have applied four different band-pass filters to $B \mu$ in order to separate EPBs of different scale sizes. The cut-off periods of the filters are listed in Table 1. For example, in the smallest range (1 s average low-pass filter and $5 \mathrm{~s}$ average high-pass filter) only an along-track wavelength of less than $76 \mathrm{~km}$ is considered. But if $B \mu$ is first smoothed by an $8 \mathrm{~s}$ average and then high-pass filtered with $40 \mathrm{~s}$ averages, EPB structures with wavelengths $120-608 \mathrm{~km}$ will get through.

ROCSAT-1 was launched on 27 April 1999 into a circular orbit at an altitude of $600 \mathrm{~km}$ and with an inclination of $35^{\circ}$. It sampled the dip equator at all local times in 25 days. Based on the measurements taken by the Ionospheric Plasma and Electro-dynamic Probe Instrument (IPEI) on board the satellite, Fejer et al. (2008) constructed a climatological model of the equatorial F-region vertical plasma drift. In this paper, we have used the same vertical drift data from July 1999 to June 2004 but resampled them.

Similar to FGM data, the vertical drift is only used from periods of geomagnetically quiet conditions $\left(K_{p} \leq 3.5\right)$. These data were grouped for every month into overlapping 2 month bins and sorted into overlapping $30^{\circ}$ wide longitude bins. The local time (LT) distribution is discretized into 30 min steps derived from overlapping 1-h local time bins except for the 17:00-22:00 LT sector, where $30 \mathrm{~min}$ bins are used and $15 \mathrm{~min}$ resolution is obtained, in order to ac-
Table 2. The solar flux ranges and the relationship between $S_{\mathrm{PRE}}$ and $v_{z}^{\mathrm{P}}$ for different solar flux levels.

\begin{tabular}{rrccl}
\hline $\begin{array}{r}\text { F10.7 } \\
\text { range }\end{array}$ & $\begin{array}{r}\text { Mean } \\
\text { F10.7 }\end{array}$ & $\begin{array}{c}\text { Corr. } \\
\text { coefficient }\end{array}$ & $\begin{array}{c}\text { Slope } \\
(a)\end{array}$ & $\begin{array}{l}\text { Bias } \\
(b)\end{array}$ \\
\hline $80-115$ & 98 & 0.72 & 7.41 & -16.3 \\
$100-145$ & 124 & 0.87 & 8.75 & -22.61 \\
$130-175$ & 155 & 0.94 & 9.18 & -17.20 \\
$160-205$ & 180 & 0.91 & 9.53 & -24.01 \\
\hline
\end{tabular}

count more accurately for the rapidly changing evening prereversal drift enhancements (Fejer et al., 2008). For each local time, month, and longitude bin, we have grouped the drift data into 4 solar flux bins. The F10.7 ranges of these groups and the average flux values for each group are listed in Table 2 .

\section{Observations}

The purpose of this paper is to investigate EPBs of different spatial scale sizes in the meridional plane. As described in Sect. 2, a dedicated program is applied to detect EPB events from CHAMP FGM data. Figure 1 shows two examples of EPBs encountered by CHAMP. The top panel presents electron density from CHAMP PLP data. Within the gray shaded windows, we can see electron density depletions. It indicates that the satellite encountered a series of EPBs on this orbit. The middle panel presents the variations of the magnetic field component $B \mu$, parallel to the background field, which correlates well with the electron density depletion. This is attributed to the diamagnetic effect of dense plasma (Lühr et al., 2003). The bottom panel shows the spectrum of the magnetic signature over the latitude range affected by EPBs $\left( \pm 17^{\circ}\right.$ magnetic latitude). Comparing the two EPB events, we find much more fine structure in the left frame, which is taken over Brazil than in the right frame, which has been recorded over eastern Asia. The visual impression is confirmed by the spectra of the magnetic field variations. The spectral amplitudes are about 10 times larger from the Brazilian event.

By using different band-pass filters, we confine the signals in the FGM data to highlight EPBs of different scale sizes. The event presented in Fig. 1 (left side) is used as an example to demonstrate the effect of the different band-passes. All four filters are applied to the same event and the results are shown in Fig. 2. It starts in the upper left with the largest scales, 120-608 km, and goes down to scales of about 15$76 \mathrm{~km}$ in the lower right frame. In the frame below, the time series of the filtered and rectified signal is shown. EPBs are detected based on this signal. Comparing the four time series clearly reveals that, with the longer low band-pass (LP) filter, more of the EPB fine structure period is smoothed out. Conversely, for higher frequency band-passes, the filtered and rectified signal gets smaller, and large events break up 

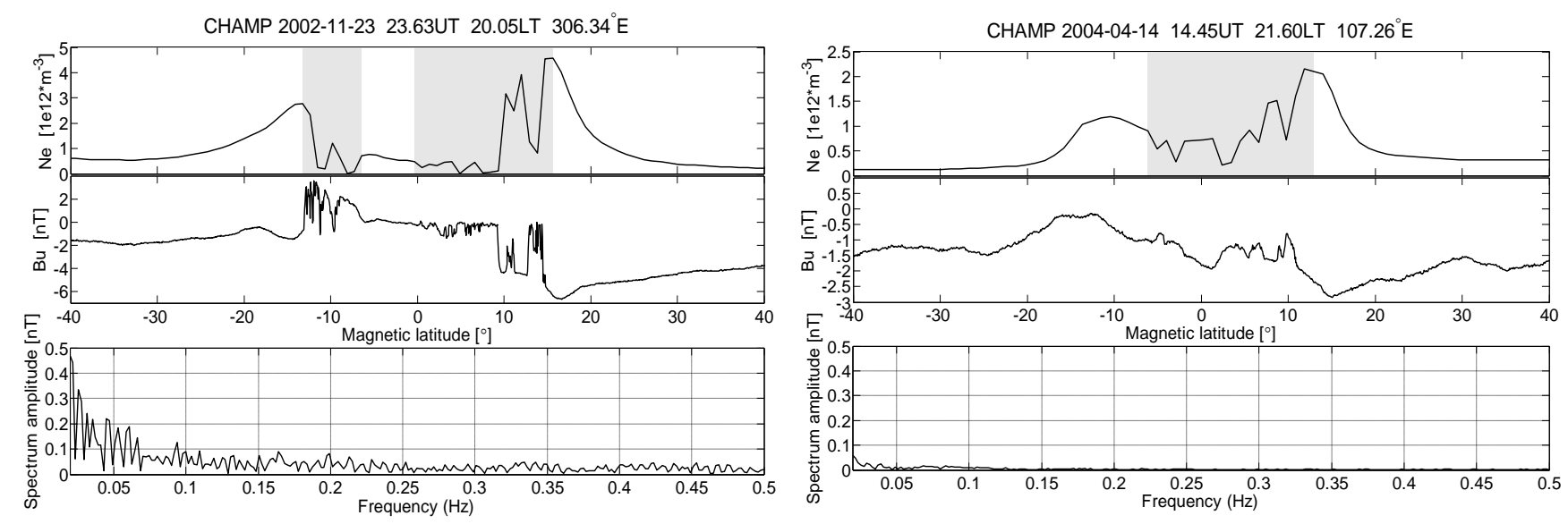

Fig. 1. Two examples of EPB detections by CHAMP. Shown are electron density changes over the pass (top), the corresponding variation of the parallel component, $B \mu$ (middle) and the spectrum of $B \mu$ (bottom) for two EPB events.
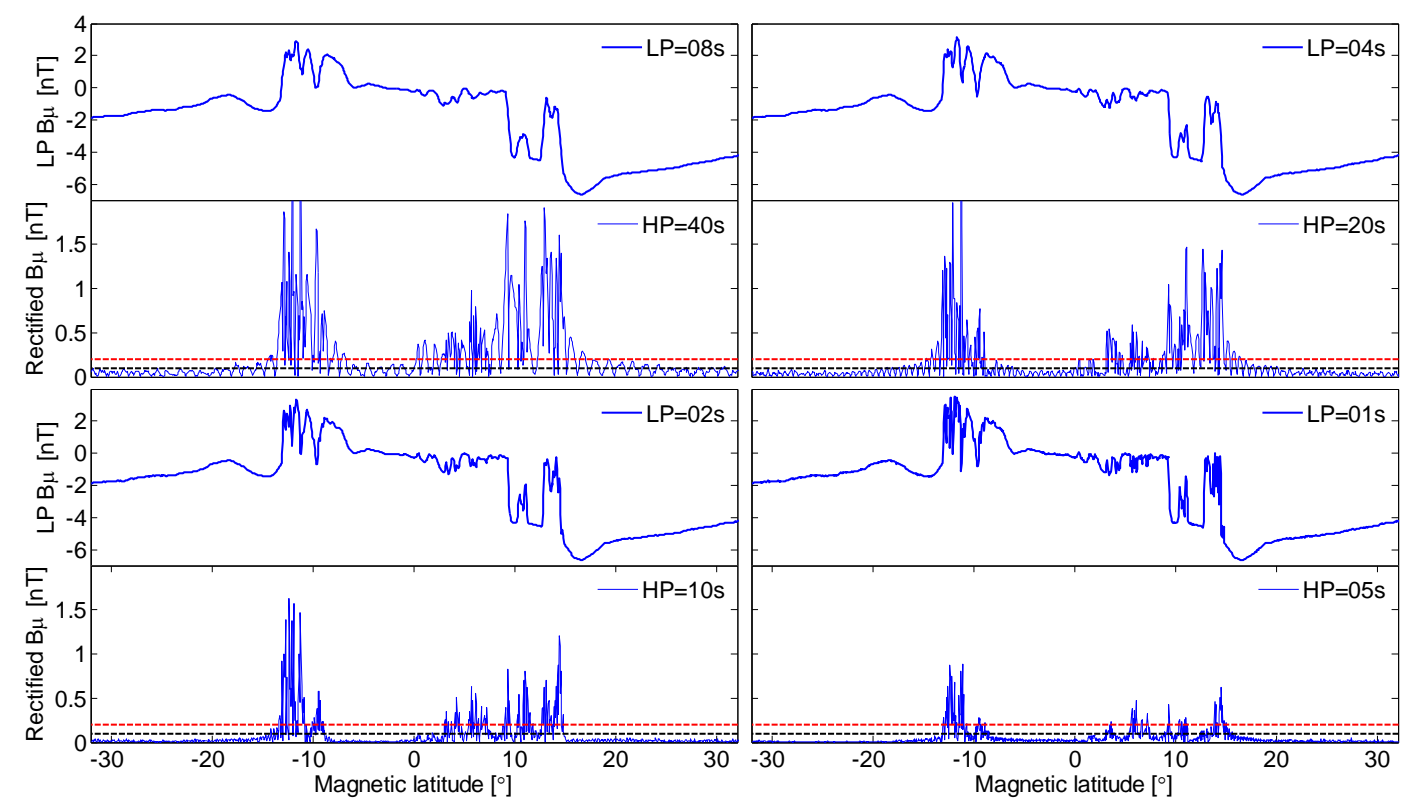

Fig. 2. Time series of the parallel magnetic field component $B \mu$ from Fig. 1, middle panel, after applying different low-pass filters (upper panels); high-pass filtered and rectified signals (lower panels). The red and black dashed lines in the lower panels represent the upper and lower thresholds applied for the automatic EPB detection process.

into groups of shorter scale. The small-scale EPBs exhibit the largest amplitudes at the poleward edges.

As has been shown in many previous papers, the largest occurrence rates of EPBs are observed above Brazil around December solstice months (e.g. Burke et al., 2004; Stolle et al., 2006; Su et al., 2008; Xiong et al., 2010). Figure 3 shows the longitudinal/seasonal (S/L) distribution of EPBs detected by the method described above, separately for the four bandpass filter groups. Detected EPBs are sorted into bins of 1 month and $10^{\circ}$ in longitude for the determination of the occurrence rates. Rates have been determined by dividing the number of passes with positive detections by the total num- ber of orbits passing over that longitude bin. For all four different band-pass filters, the largest occurrence rates are found between $300-340^{\circ}$ longitudes around November and December. Otherwise the occurrence rates are severely reduced towards smaller scales. Only for the largest scales we obtain a $\mathrm{S} / \mathrm{L}$ distribution similar to previous studies. It is worth noting that the mean value of the solar flux prevailing during days of positive detections is increasing gradually from large-scale to small-scale events. We obtain average F10.7 values of 159 , 167,171 and 176 for the four filter groups, respectively. This indicates that for the more structured EPBs to reach the altitude of CHAMP a higher solar flux level is needed. 


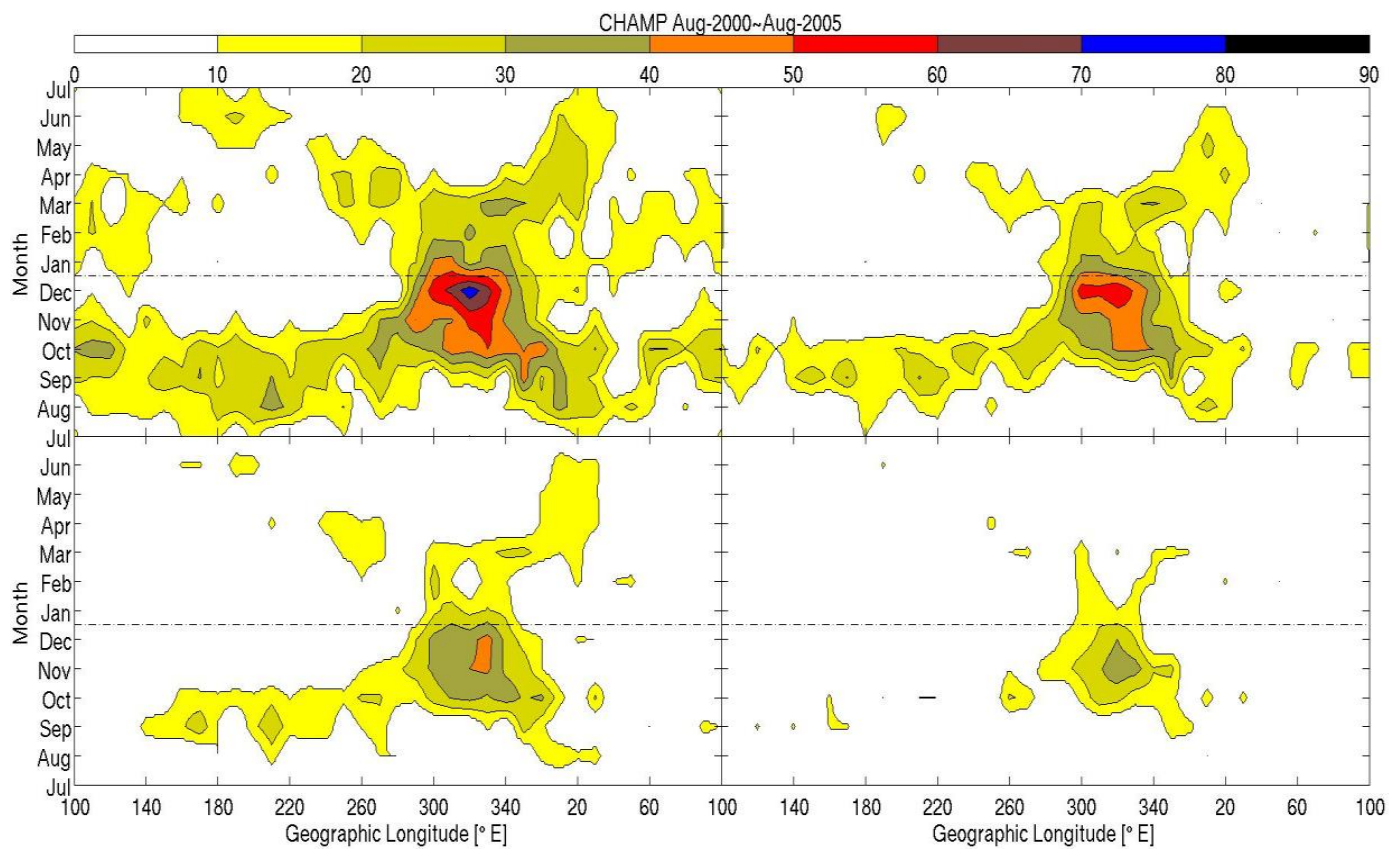

Fig. 3. The seasonal/longitudinal distribution of EPBs occurrence rates for different meridional scale size, using different band-pass filters: $\mathrm{LP}=8 \mathrm{~s}, \mathrm{HP}=40 \mathrm{~s}$ (top left); $\mathrm{LP}=4 \mathrm{~s}, \mathrm{HP}=20 \mathrm{~s}$ (top right); $\mathrm{LP}=2 \mathrm{~s}, \mathrm{HP}=10 \mathrm{~s}$ (bottom left); LP $=1 \mathrm{~s}, \mathrm{HP}=5 \mathrm{~s}$ (bottom right).

In order to prove this suggestion we have also investigate the vertical plasma drift, $V_{Z}$, data of ROCSAT-1. Su et al. (2008) and Stolle et al. (2008) have shown that EPB occurrence rates show a good correlation with the peak vertical plasma drift during the pre-reversal enhancement, $V_{Z}^{\mathrm{P}}$. But the correlation between EPB occurrence rate and the integrated vertical velocity, $S_{\mathrm{PRE}}$, is even higher. Following the approach of Stolle et al. (2008) we get

$S_{\mathrm{PRE}}=\int_{t_{1}}^{t_{2}} V_{Z} \cdot \mathrm{d} t$

where $t_{1}$ is the local time with the first occurrence of upward plasma drift (positive value of $V_{Z}$ ) after the afternoon local minimum (between noon and postsunset maxima), and $t_{2}$ is the time of polarity switch in vertical plasma drift from upward to downward. S SRE contains both information about the vertical plasma drift velocity and the extend to which the plasma is lifted up. At greater height the ion/neutral collision frequency is reduced. Therefore, the growth rate of instabilities is larger (Ossakow, 1981). Stolle et al. (2008) have identified $S_{\mathrm{PRE}}$ as the preferred indicator for EPB generation. In order to find the relation between $S_{\mathrm{PRE}}$ and the vertical plasma drift we have correlated the two quantities separately for four solar flux ranges. Results of the analysis are listed in Table 2. The highest correlation coefficient, 0.94, is obtained for the solar flux range F10.7 $=130-175$ sfu. Figure 4 shows a scatter plot of $V_{Z}^{\mathrm{P}}$ versus $S_{\mathrm{PRE}}$. All the points are reasonably well grouped around the regression line. The linear regression has the form $S_{\mathrm{PRE}}=9.18 \cdot V_{Z}^{\mathrm{P}}-17.20$ where $V_{Z}^{\mathrm{P}}$ is

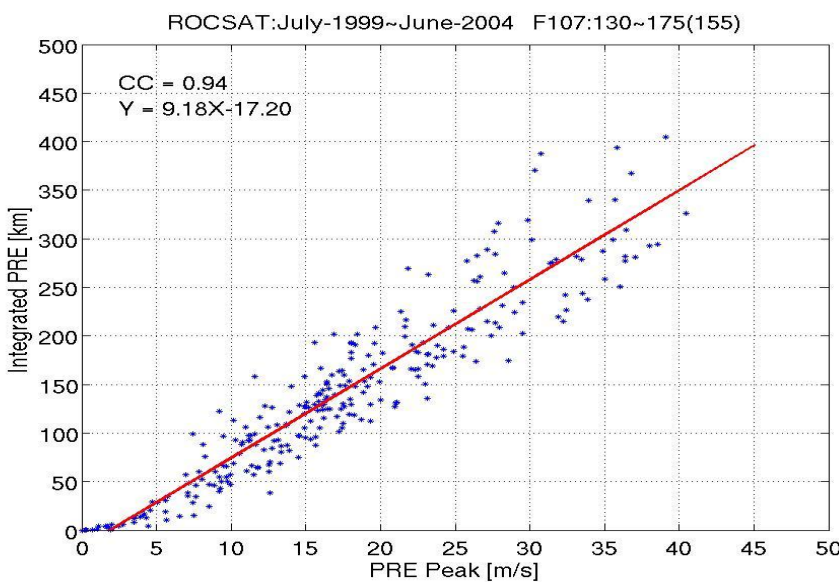

Fig. 4. The relationship between the peak value of the vertical plasma drift velocity and the integrated vertical plasma drift velocity for the solar flux range F10.7 = 130-175 (average F10.7 = 155)

given in $\left(\mathrm{m} \mathrm{s}^{-1}\right)$ and $S_{\mathrm{PRE}}$ in $(\mathrm{km})$. As can be seen from Table 2, the slope of the regression line increases when higher solar flux ranges are considered.

In order to inspect the relation of EPB occurrences with the size of $S_{\mathrm{PRE}}$, we have plotted the distribution of the integrated vertical velocity. From Fig. 5 we can see that the integrated vertical plasma drift velocity shows a similar S/L distribution as the EPB occurrence rate. $S_{\mathrm{PRE}}$ reaches almost $500 \mathrm{~km}$ above the Brazilian region around November, where the most small-scale EPBs are detected, as is evident from 


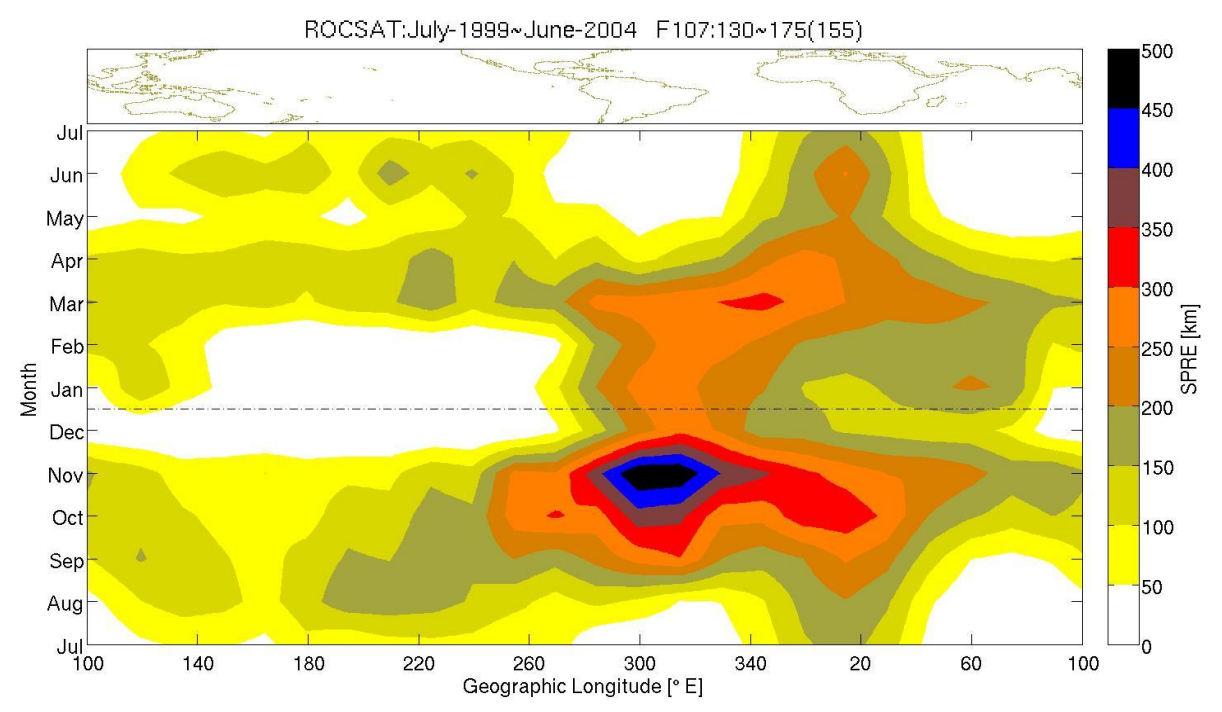

Fig. 5. The seasonal/longitudinal distribution of the integrated vertical plasma drift velocity.

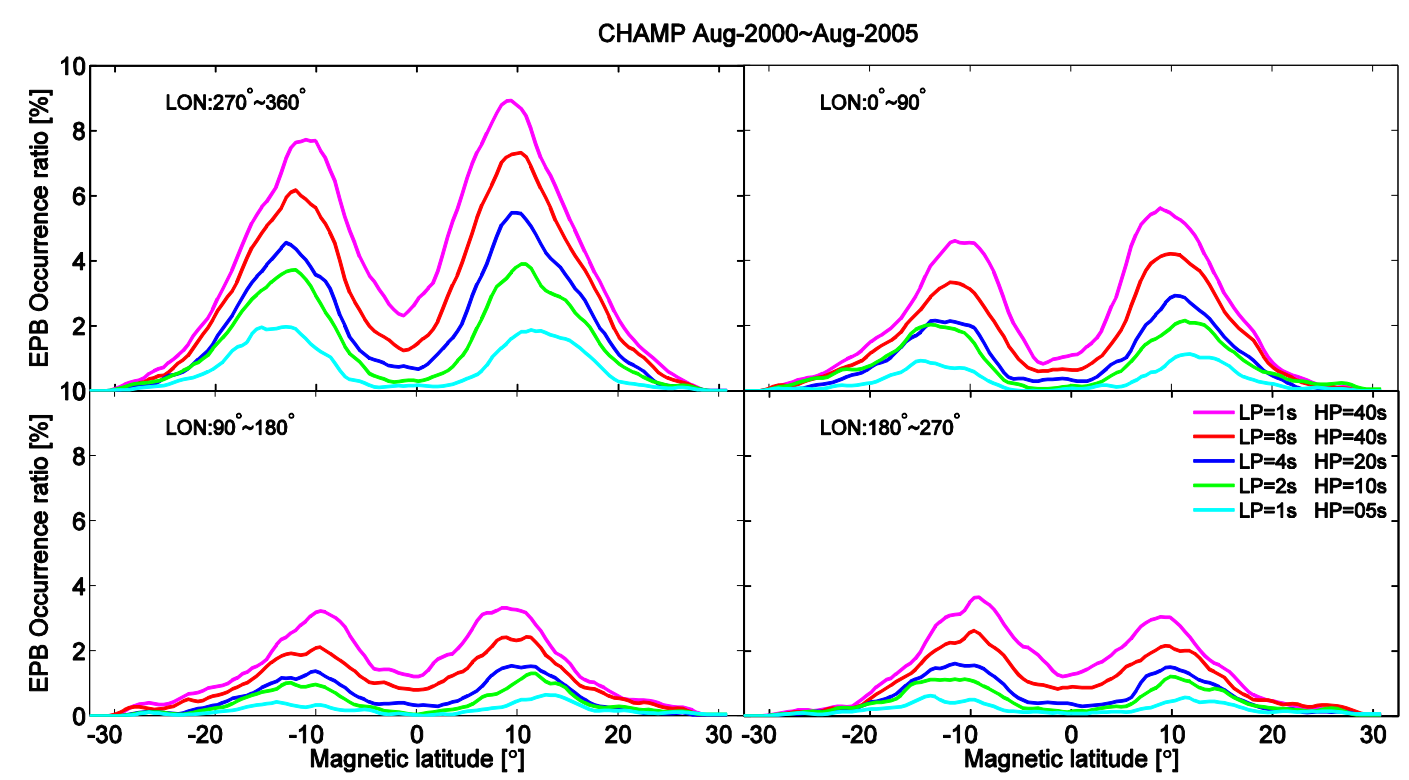

Fig. 6. Latitudinal distribution of the EPB occurrence rate from the different band-passes separately for four longitude sectors.

Fig. 2. It seems that for a given solar flux level, the F-layer in this region is easier to be lifted to higher altitude, where the plasma is obviously more susceptible to the generation of small scale size EPBs.

In a next step we investigate the latitudinal distribution of the EPB occurrence rate following the approach as described by Xiong et al. (2010). EPB signals are sorted into $1^{\circ}$ wide latitude bins. Count numbers in each bin are normalized by the total number of satellite passes over that bin. From Fig. 6 we can see that all the occurrence rate profiles from the different filters show two symmetric peaks, one north and one south of the magnetic equator. In order to obtain a more quantitative description of the distribution, we have fitted a Gaus- sian function separately to each of the peaks. The function is specified as

$y=A \cdot \exp \left(-\frac{\left(x-x_{0}\right)^{2}}{2 \sigma^{2}}\right)$

where $A$ is the peak occurrence rate amplitude, $x_{0}$ is the latitude of the peak and $\sigma$ is the width in degrees of latitude of the distribution. All pairs of distribution peaks are almost symmetrical around the dip equator. The results of the fitting procedure are listed in Table 3. We find a general trend that the more structured EPBs detected at CHAMP altitude has been lifted up higher than the large-scale bubbles. The number of EPB detections differs significantly between the four 
Table 3. Parameters of Gaussian fits to the latitude profiles of the EPB occurrence rates separately for four longitude sectors (see Fig. 5).

\begin{tabular}{lcccccccc}
\hline Longitude Sectors $\left(^{\circ}\right)$ & HP $(\mathrm{s})$ & \multicolumn{3}{c}{ Southern Hemisphere } & \multicolumn{3}{c}{ Northern Hemisphere } & \multirow{2}{*}{ Apex height $(\mathrm{km})$} \\
\cline { 2 - 7 } & & $A(\%)$ & $X_{0}\left({ }^{\circ}\right)$ & $\sigma\left(^{\circ}\right)$ & $A(\%)$ & $X_{0}\left({ }^{\circ}\right)$ & $\sigma\left(^{\circ}\right)$ & \\
\hline $270-360$ & 40 & 5.53 & -11.67 & 6.15 & 6.5 & 11.68 & 6.30 & 689 \\
& 20 & 3.84 & -12.10 & 5.75 & 4.79 & 12.07 & 5.65 & 710 \\
& 10 & 3.43 & -13.35 & 5.07 & 3.34 & 12.97 & 5.35 & 770 \\
& 5 & 1.87 & -13.85 & 4.61 & 1.37 & 13.75 & 5.44 & 808 \\
\hline $0-90$ & 40 & 2.70 & -12.05 & 6.30 & 3.65 & 11.99 & 5.85 & 706 \\
& 20 & 1.75 & -12.26 & 5.82 & 2.49 & 12.14 & 4.39 & 716 \\
& 10 & 1.64 & -13.65 & 5.73 & 1.48 & 13.14 & 5.93 & 783 \\
& 5 & 0.75 & -13.66 & 4.83 & 0.73 & 13.89 & 5.44 & 806 \\
\hline $90-180$ & 40 & 1.54 & -9.50 & 8.68 & 1.84 & 10.64 & 7.98 & 613 \\
& 20 & 1.13 & -10.71 & 5.98 & 1.06 & 11.40 & 6.43 & 658 \\
& 10 & 0.71 & -12.63 & 6.32 & 0.71 & 13.73 & 5.99 & 771 \\
\hline $180-270$ & 5 & 0.24 & -13.08 & 7.44 & 0.32 & 14.58 & 5.63 & 810 \\
& 40 & 2.25 & -9.64 & 5.79 & 1.45 & 9.50 & 8.66 & 592 \\
& 20 & 1.39 & -10.64 & 5.39 & 0.86 & 11.55 & 7.11 & 660 \\
& 10 & 0.96 & -12.56 & 5.80 & 0.69 & 14.09 & 7.00 & 779 \\
& 5 & 0.37 & -13.61 & 6.31 & 0.25 & 14.48 & 6.64 & 823 \\
\hline
\end{tabular}

longitude sectors. However, the latitude of peak occurrences is practically the same for all longitude sectors. For reference, we have added a column in Table 3 giving the apex height of fluxtubes containing the highest occurrence rate. Our observations suggest that CHAMP detects small-scale plasma irregularities only on fluxtubes that have been lifted up high enough. On fluxtubes with lower apex height, largescale structure are preferably observed at CHAMP altitude.

\section{Discussion}

The purpose of this study is to investigate the scale size of equatorial plasma bubbles and find out their specific characteristics. For achieving this goal we have chosen a statistical approach. CHAMP magnetic field and electron density data over the period August 2000 to July 2005 have been considered for this purpose. Within $5 \mathrm{yr}$ CHAMP provides an even coverage of all local times and seasons. This time interval provides, in addition, a good overlap with the vertical plasma drift measurements from ROCSAT-1 (July 1999 to June 2004). Furthermore, these years coincide with the high activity period of solar cycle 23 .

For the investigation of the EPB structural scales, we make use of the high-resolution CHAMP magnetic field data. This is done because the electron density is sampled only once per $15 \mathrm{~s}$, corresponding to one sample per $114 \mathrm{~km}$. The relation between plasma density and magnetic field variations can be explained by the diamagnetic effect of dense plasmas. According to Lühr et al. (2003), changes in total field strength, $\Delta B$, can be approximated by
$\Delta B=\Delta n \cdot\left(T_{\mathrm{e}}+T_{\mathrm{i}}\right) \cdot \frac{k}{B}$

where $n$ is the electron density, $T_{\mathrm{e}}$ and $T_{\mathrm{i}}$ are the electron and ion temperatures, $k$ is the Boltzmann constant and $B$ the ambient magnetic field strength. Here, we assume that the temperatures are about the same inside and outside the bubbles. It is obvious that the ambient magnetic field strength influences the scaling factor between $\Delta n$ and $\Delta B$.

For discriminating bubbles of different spatial scales, we have applied four band-pass filters to the magnetic field data. Due to the polar orbit of CHAMP, we obtain only information about scale length in the meridional plane. The shortest filter considers sizes up to $76 \mathrm{~km}$, the longest up to $608 \mathrm{~km}$. Extending the high pass filter to longer periods (larger scales) causes a severe contamination of the small EPB magnetic signals by magnetic pulsations. We found out that the EPB occurrence distribution derived from plasma density measurement (e.g. Xiong et al., 2010) is reproduced best by magnetic field data, when the $1 \mathrm{~Hz}$ samples are high pass filtered with a cut off period of $40 \mathrm{~s}$ ( $\sim 608 \mathrm{~km}$ scale size $)$. For completeness it is noted that our detection algorithm is based on a fixed $\Delta n$ (see Xiong et al., 2010), opposed to $\Delta n / n$ as used in many other studies (e.g. Burke et al., 2004; Su et al., 2006).

\subsection{Occurrence distribution of EPBs}

When talking about the EPB characteristics presented here, we have to keep in mind that they are valid only for the orbital altitude of CHAMP $(\sim 400 \mathrm{~km})$. The satellite may cut through the topside or bottom side of an irregular region in 


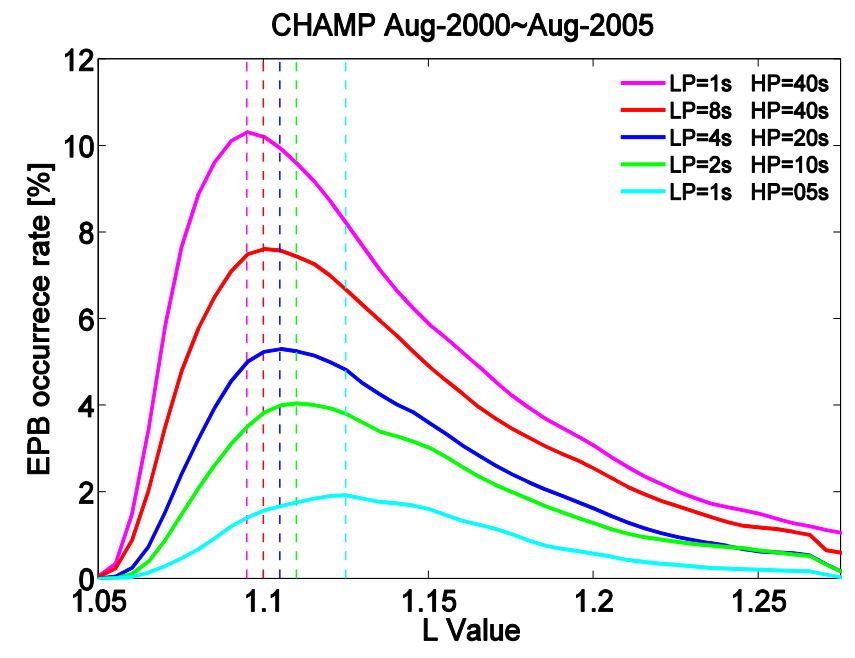

Fig. 7. L-value distribution of fluxtubes on which EPB of different scale size where detected.

the meridional plane depending on the mean altitude of the bubble structure. From Fig. 3, comparing the top left panel to the right bottom panel, one can see that there are many more large scale EPBs at CHAMP attitude. An appreciable amount of smallest scale $(15-76 \mathrm{~km})$ bubbles is only detected in the longitude sector $270-360^{\circ}$ around December solstice months. The four S/L distributions in Fig. 3 are all selfsimilar, just the percentages change from frame to frame. $\mathrm{Ob}-$ viously, the small scales are substructures of large-scale bubbles. Conversely, we observe often large-scale EPBs without small structures (see Fig. 1, right example).

Another interesting fact is the large apex height of the fluxtubes containing small-scale features. From Fig. 6 we can deduce that the occurrence rates of highly structured events are quite different for the four considered longitude sectors. However, it is interesting to note that CHAMP detects at all longitudes the small structures preferably on fluxtubes with apex heights of about $800 \mathrm{~km}$. Similarly for the other scale sizes, we find comparable apex heights at all longitudes for the peak occurrence rates. Over the $5 \mathrm{yr}$ considered the CHAMP altitude has become lower. In order to account for that we computed the L-value of the fluxtube whenever detecting an EPB. Results are presented in Fig. 7. Here again the poleward shift of the occurrence peaks for smaller EPB structures is evident. The smallest scale attains highest rates at $L=1.125 R_{\mathrm{E}}$ which corresponds to an apex height of about $800 \mathrm{~km}$. These observations suggest that an irregular region is highly structured at its bottom side and that depletion features may get larger towards apex. Support for this suggestion is provided by Aveiro and Hysell (2010) who successfully modeled a plasma irregularity structure using a 3-dimensional numerical simulation. They applied two kinds of instabilities to obtain high altitude plasma irregularities. At the bottom side of the ionosphere between about 200 and $250 \mathrm{~km}$, the irregularity was driven by the collisional shear instability (CSI). Also after several minutes of simulation run time, the effect of CSI did not reach $\mathrm{F}$ region peak altitudes, but remained at the bottom side. CSI was found to cause larger relative amplitudes than structures created by the generalized Rayleigh-Taylor (GRT) instability. The latter instability is more effective at altitudes near the F peak where the vertical plasma density gradient is less steep than at the bottom side. GRT structures have been found to extent also to higher altitudes, and "they do not appear to be very turbulent" (Aveiro and Hysell, 2010). Simulation runs combining CSI and GRT produced structures within the F-region at nearly all altitudes and scale sizes. This implies that welldeveloped irregularities include also smaller scale structures. This is consistent with our observations that only the high altitude bubbles contain the smallest detected scales.

\subsection{Influence of the vertical plasma drift}

It is known that the ionospheric F-region is uplifted considerably after sunset by the strong vertical plasma drift during the pre-reversal enhancement (PRE). This makes the F-region bottom side more susceptible to initiating the RayleighTaylor instability (Kelley, 2009, Sect. 4). Stolle et al. (2008) have confirmed in a statistical study the close relation of EPB occurrence rate with the value of the integrated verti-

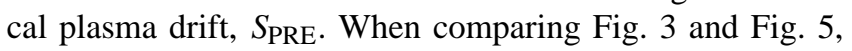
we can clearly confirm the conclusions of Stolle et al. (2008) that the EPB occurrence has a higher correlation with $S_{\text {PRE. }}$. Particularly large values of $S_{\text {PRE }}$ up to $500 \mathrm{~km}$ are found in the Brazilian sector during the months around November. Here, we find irregularities on fluxtubes reaching on average up to $800 \mathrm{~km}$ at their apex. This is exactly the location and time where the majority of small-scale EPBs are detected. All this points in the direction of an especially high uplift of the F-region. On top of that we found on average a higher solar flux level prevailing during days of smallscale EPBs $(\mathrm{F} 10.7=176 \mathrm{sfu})$ than during large scale EPBs $(\mathrm{F} 10.7=159 \mathrm{sfu})$ detections. This adds further evidence to the connection between small-scale structures and a high Fregion. It is well known that the vertical plasma velocity during PRE is closely controlled by the solar flux level and with that it directly influences $S_{\text {PRE }}$ (see Fig. 4). At first order we may interpret $S_{\text {PRE }}$ values as the amount of plasma uplift. For detecting small-scale EPB we need $S_{\text {PRE values larger }}$ than $400 \mathrm{~km}$. This brings the fluxtube up to $800 \mathrm{~km}$ and enables CHAMP to detect highly structured features at $400 \mathrm{~km}$ altitude. CHAMP detects large-scale EPBs on flux tubes that reach on average apex heights of $600 \mathrm{~km}$, and for those $S_{\mathrm{PRE}}$ values of $S_{\mathrm{PRE}}>100 \mathrm{~km}$ are sufficient.

Our preferred interpretation of the obtained results is schematically illustrated in Fig. 8. Post-sunset plasma irregularities develop along fluxtubes. Small scale structures form at the lower end and higher up features become larger. Depending on the integrated vertical plasma drift CHAMP is sampling the irregularity either near the top or close to the 


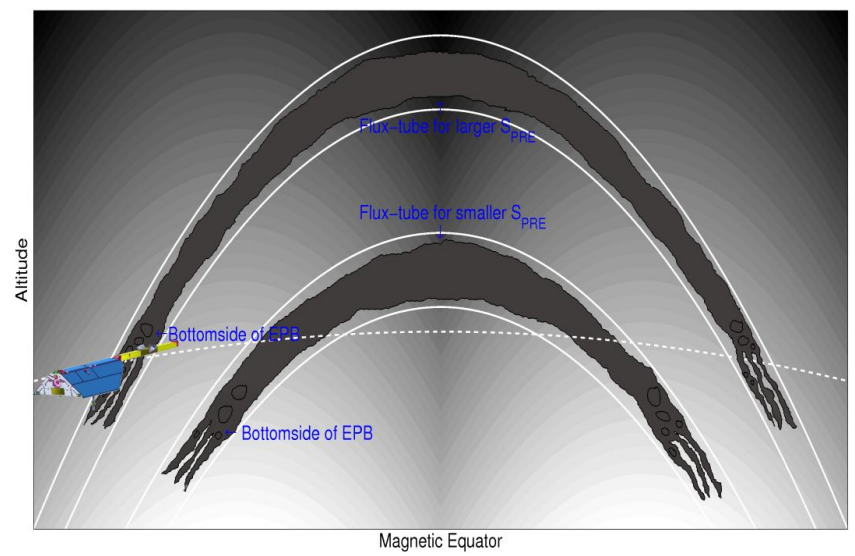

Fig. 8. Carton illustrating the structure of plasma irregularities relative to CHAMP orbit height.

bottom. In case of fluxtubes reaching up to $800 \mathrm{~km}$, the small structures are observed $400 \mathrm{~km}$ lower. This may be regarded as indications for the vertical extend of EPBs. Since the majority of EPBs reach an apex height of $600 \mathrm{~km}$, the majority of small features may be expected at $200-300 \mathrm{~km}$ altitude. However, owing to our single satellite observations, we cannot say whether the fine structure is limited to the lower part of EPBs or is present at all altitudes.

\subsection{Local time dependence of EPB structures}

CHAMP on its polar orbit provides only meridional cuts though the plasma irregularities. Longitudinal (local time) variations can only be investigated by statistical methods. In order to check that, we determined the local time dependence of the occurrence rate. Assuming no dependence on longitude, we limited the investigation to the longitude sector $270-360^{\circ} \mathrm{E}$, where the majority of the small-scale EPBs is observed. Figure 9 shows the EPB occurrence rate versus local time (LT) for the hours 19:00-01:00 LT separately for the four filter bands. As seen before, more events are detected by longer period filters. Up to 22:00 LT rates are increasing. Since the ratio between large and small structures stays about the same, between 19:30 and 22:00 LT, we find no indication of a significant relative increase of the smallscale structures up to 22:00 LT. After 22:00 LT, the situation is different. At that time we can expect a downward plasma drift during high and moderate solar activity times (Fejer et al., 2008). While the occurrence of large-scale features stays almost constant until midnight, the small-scale structures decay rapidly. These observations suggest following conclusions: (1) The growth of well-developed plasma irregularities is confined until about 22:00 LT. Small and large scales develop at the same rate. Due to the increase in small scales, we expect that the structures are uplifted mainly in this LT sector. (2) After 22:00 LT, when the vertical plasma drift is

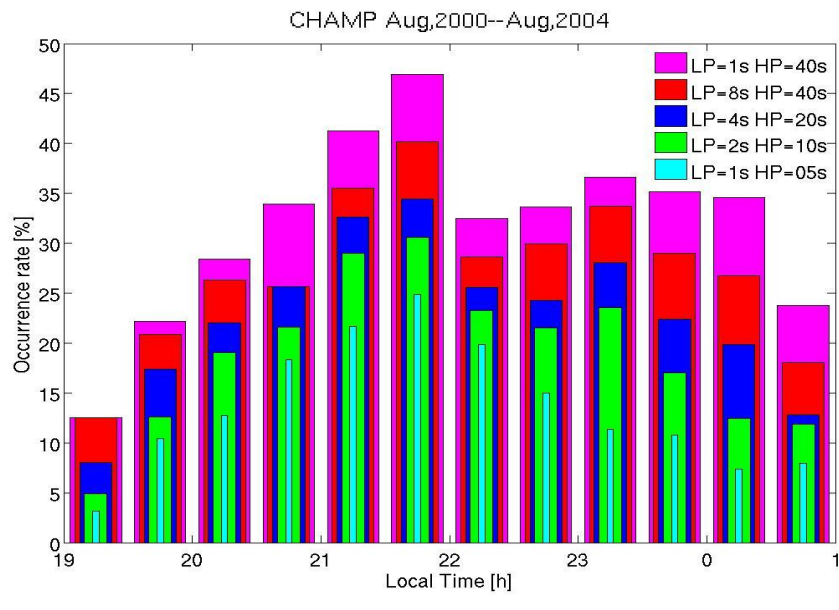

Fig. 9. Local time distribution of EPB occurrence rates for different scale sizes at 270-360 degree longitude.

mainly downward stabilizing the ionosphere, we observe that smaller scales decay much faster than large scale structures.

From the data available, we can say that we detected smallscale bubbles, when the corresponding L-shell reached to high apex altitudes, but we do not know whether EPBs on low fluxtubes have also small-scale structures at the bottom side of the bubble, below the sampling altitude of CHAMP.

\section{Summary}

In this paper we have presented results on the characteristics of plasma irregularities of different scale sizes. The studies are based on CHAMP observations from the high solar activity years August 2000-August 2005. Main features deduced are as follows:

1. CHAMP detects many more EPBs with scale sizes 120 $608 \mathrm{~km}$ than $15-76 \mathrm{~km}$. Small-scale EPBs at CHAMP altitude are confined in general to the longitude sector $270-360^{\circ} \mathrm{E}$ and the months around November.

2. There is a good correlation between the integrated vertical plasma drift after sunset and the detected scale size of EPB structures. CHAMP detects structured EPBs only in regions of high ionospheric uplift.

3. Highest occurrence rates are encountered on fluxtubes that reach apex heights of $800 \mathrm{~km}$. Large-scale EPBs exhibit maximum occurrence rates on fluxtubes with apex heights of about $600 \mathrm{~km}$.

4. The occurrence rates increase steadily with local time, from 19:00 LT up to 22:00 LT, at a comparable rate for all scale sizes. Then the small-scale features decay faster than the large-scale EPBs. During the early evening the general uplift of the irregular region seems to bring more events into the height range of CHAMP as 
time progresses. The reason for the fast decay of small structures after 22:00 LT is suggested to be related to the downward plasma drift, but this cannot be determined uniquely from our observations.

5. Based on the interpretation of model simulations by Aveiro and Hysell (2010), we suggest that the bottom side of plasma irregularities is assigned to smaller scale structures than those at apex height, and that irregularities which are well developed in altitude have structures to very low scales (according to CHAMP data down to kilometer-scale).

The results obtained here should be verified by multi-satellite approaches and numerical modeling to uniquely assess the height distribution of bubble structures.

Acknowledgements. The CHAMP and GRACE missions are sponsored by the Space Agency of the German Aerospace Center (DLR) through funds of the Federal Ministry of Economics and Technology. We would like to thank the German Space Operations Center (GSOC) of the German Aerospace Center (DLR) for providing continuously and nearly $100 \%$ of the raw telemetry data of the CHAMP and GRACE satellites. One of the authors (Chao Xiong) is supported by the German Academic Exchange Service (DAAD) and China Scholarship Council (CSC).

The service charges for this open access publication have been covered by a Research Centre of the Helmholtz Association.

Topical Editor M. Pinnock thanks T. Yokoyama and one anonymous referee for their help in evaluating this paper.

\section{References}

Aveiro, H. C. and Hysell, D. L.: Three dimensional numerical simulation of equatorial $\mathrm{F}$ region plasma irregularities with bottomside shear flow, J. Geophys. Res., 115, A11321, doi:10.1029/2010JA015602, 2010.

Burke, W. J., Gentile, L. C., Huang, C. Y., Valladares, C. E., and Su, S. Y.: Longitudinal variability of equatorial plasma bubbles observed by DMSP and ROCSAT-1, J. Geophys. Res., 109, A12301, doi:10.1029/2004JA010583, 2004.

Farley, D., Balsey, B., Woodman, R., and McClure, J.: Equatorial Spread F: Implications of VHF Radar Observations, J. Geophys. Res., 75, 7199-7216, 1970.

Fejer, B. G., Scherliess, L., and de Paula, E. R.: Effects of the vertical plasma drift velocity on the generation and evolution of equatorial spread F, J. Geophys. Res., 104, 19859-19869,, 1999.

Fejer, B. G., Jensen, J. W., and Su, S.-Y.: Quiet time equatorial $\mathrm{F}$ region vertical plasma drift model derived from ROCSAT-1 observations, J. Geophys. Res., 113, A05304, doi:10.1029/2007JA012801, 2008.

Huang, C. Y., Burke, W. J., Machuzak, J. S., Gentile, L. C., and Sultan, P.: DMSP observations of equatorial plasma bubbles in the topside ionosphere near solar maximum, J. Geophys. Res., 106, 8131-8142, 2001.

Hysell, D. L.: A review and synthesis of plasma irregularities in equatorial spread F, J. Atmos. Sol.-Terr. Phys., 62, 1037-1056, 2000.
Hysell, D. L. and Seyler, C. E.: A renormalization group approach to estimation of anomalous diffusion in the unstable equatorial F region, J. Geophys. Res., 103, 26731-26737, doi:10.1029/98JA02616, 1998.

Jayachandran, B., Balan, N., Rao, P. B., Sastri, J. H., and Bailey, G. J.: HF Doppler and ionosonde observations on the onset conditions of equatorial spread-F, J. Geophys. Res., 98, 13741-13750, 1993.

Kelley, M. C.: The Earth's Ionosphere, Plasma Physics and Electrodynamics, 2nd Edn., Academic, San Diego Calif, 2009.

Lühr, H., Rother, M., Maus, S., Mai, W., and Cooke, D.: The diamagnetic effect of the equatorial Appleton anomaly: Its characteristics and impact on geomagnetic field modeling, Geophys. Res. Lett., 30, 1906, doi:10.1029/2003GL017407, 2003.

Makela, J. J. and Kelley, M. C.: Using the 630.0-nm nightglow emission as a surrogate for the ionospheric Pedersen conductivity, J. Geophys. Res., 108, 1253, doi:10.1029/2003JA009894, 2003.

Makela, J. J., Kelley, M. C., and Su, S.-Y.: Simultaneous observations of convective ionospheric storms: ROCSAT1 and ground-based imagers, Space Weather, 3, S12C02, doi:10.1029/2005SW000164, 2005.

Ossakow, S. L.: Spread F theories - A review, J. Atmos. Sol.-Terr. Phys., 43, 437-452, 1981.

Reigber, C., Lühr, H., and Schwintzer, P.: CHAMP mission status, Adv. Space Res., 30, 129-134, 2002.

Singh, S., Johnson, F. S., and Power, R. A.: Gravity wave seeding of equatorial plasma bubbles, J. Geophys. Res., 102, 7399-7410, doi:10.1029/96JA03998, 1997.

Sobral, J. H. A., Abdu, M. A., Takahashi, H., Sawant, H., Zamlutti, C. J., and Borba, G. L.: Solar and geomagnetic activity effects on nocturnal zonal velocities of ionospheric plasma depletions, Adv. Space Res., 24, 1507-1510, 1999.

Stolle, C., Lühr, H., Rother, M., and Balasis, G.: Magnetic signatures of equatorial spread $\mathrm{F}$, as observed by the CHAMP satellite, J. Geophys. Res., 111, A02304, doi:10.1029/2005JA011184, 2006.

Stolle, C., Lühr, H., and Fejer, B. G.: Relation between the occurrence rate of ESF and the equatorial vertical plasma drift velocity at sunset derived from global observations, Ann. Geophys., 26, 3979-3988, doi:10.5194/angeo-26-3979-2008, 2008.

Su, S.-Y., Liu, C. H., Ho, H. H., and Chao, C. K.: Distribution characteristics of topside ionospheric density irregularities: Equatorial versus midlatitude regions, J. Geophys. Res., 111, A06305, doi:10.1029/2005JA011330, 2006.

Su, S.-Y., Chao, C. K., Liu, C. H., and Ho, H. H.: Meridional wind effect on anti-solar activity correlation of equatorial density irregularity distribution, J. Geophys. Res., 112, A10305, doi:10.1029/2007JA012261, 2007.

Su, S.-Y., Chao, C. K., and Liu, C. H.: On monthly/seasonal/ longitudinal variations of equatorial irregularity occurrences and their relationship with the postsunset vertical drift velocities, J. Geophys. Res., 113, A05307, doi:10.1029/2007JA012809, 2008.

Tsunoda, R. T.: Magnetic-field-aligned characteristics of plasma bubbles in the nighttime equatorial ionosphere, J. Atmos. Terr. Phys., 42, 743-752, 1980.

Whalen, J. A.: An equatorial bubble: Its evolution observed in relation to bottom side spread F and to the Appleton anomaly, J. Geophys. Res., 105, 5303-5315, 2000. 
Xiong, C., Park, J., Lühr, H., Stolle, C., and Ma, S. Y.: Comparing plasma bubble occurrence rates at CHAMP and GRACE altitudes during high and low solar activity, Ann. Geophys., 28, 1647-1658, doi:10.5194/angeo-28-1647-2010, 2010.

Yokoyama, T., Su, S.-Y., and Fukao, S.: Plasma blobs and irregularities concurrently observed by ROCSAT-1 and equatorial atmosphere radar, J. Geophys. Res., 112, A05311, doi:10.1029/2006JA012044, 2007.
Zargham, S. and Seyler, C. E.: Collisional and Inertial Dynamics of the Ionospheric Interchange Instability, J. Geophys. Res., 94, 9009-9027, doi:10.1029/JA094iA07p09009, 1989. 